\title{
A RELIGIOSIDADE/ESPIRITUALIDADE COMO RECURSO NO ENFRENTAMENTO DA COVID-19
}

\section{RELIGIOSITY/SPIRITUALITY AS A RESOURCE TO FACE COVID-19}

\section{RELIGIOSIDAD/ESPIRITUALIDAD COMO RECURSO PARA HACER FRENTE A COVID-19}

\author{
Fabio Scorsolini-Comin ${ }^{1}$, Lucas Rossato ${ }^{2}$, Vivian Fukumasu da Cunha ${ }^{3}$, Marta Regina Gonçalves Correia-Zanini ${ }^{4}$, Sandra Cristina Pillon ${ }^{5}$
}

\section{RESUMO}

Objetivo: problematizar de que modo a Religiosidade/Espiritualidade (R/E) pode ser empregada como um recurso no enfrentamento da pandemia da COVID-19. Método: Reflexão teórica baseada na literatura científica da área. Resultados: Entre as principais aplicações dessa dimensão no contexto de atenção em saúde destaca-se a R/E como recurso para a compreensão dos efeitos da pandemia na vida cotidiana; como recurso de enfrentamento e fonte de apoio para pessoas adoecidas, cuidadores e familiares; como suporte nas situações de isolamento social e quarentena; como recurso para profissionais de saúde diretamente envolvidos no combate à pandemia; como recurso na compreensão de situações de luto. Conclusão: Recomenda-se que a R/E possa ser empregada como um recurso não apenas na explicação das repercussões emocionais da doença, mas como estratégia que possa, de fato, promover um cuidado mais humano e integrado diante de um cenário pandêmico impermanente.

Palavras-chave: Pandemias; Religião; Espiritualidade; Infecções por Coronavirus.

\section{ABSTRACT}

Objective: Considering that religiosity/spirituality $(R / S)$ has been an important vertex of health care, the objective of this study is to discuss how R/S can be used as a resource to face the COVID-19 pandemic. Method: Theoretical reflection based on the scientific literature of the area. Results: Among the main applications of this dimension in the context of health care, we highlight: $R / S$ as a resource for understanding the effects of the pandemic on daily life; as a coping resource and source of support for sick people, caregivers and family members; as support in situations of social isolation and quarantine; as a resource for front-line health professionals during the pandemic; as a resource for understanding grieving situations. Conclusion: It is recommended that $\mathrm{R} / \mathrm{S}$ can be used as a resource not only in explaining the emotional repercussions of the disease, but as a strategy that can promote more humane and integrated care in the face of an impermanent pandemic scenario.

Keywords: Pandemics; Religion; Spirituality; Coronavirus Infections.

\section{RESUMEN}

Objetivo: teniendo en cuenta que la religiosidad/espiritualidad (R/E) ha sido un aspecto importante de la atención en salud, el objetivo de este estudio es analizar como R/E puede utilizarse como un recurso para hacer frente a la pandemia de COVID-19. Método: reflexión teórica basada en la literatura científica del área. Resultados: entre las principales aplicaciones de esta dimensión en el contexto de la atención en salud, destacamos: R/E como un recurso para comprender los efectos de la pandemia en la vida diaria; como recurso de enfrentamiento y punto de apoyo para personas enfermas, cuidadores y familiares; 3 ) R/E como apoyo en situaciones de aislamiento social y cuarentena; como recurso para profesionales de la salud directamente involucrados en la lucha contra la pandemia; como recurso para comprender situaciones de luto. Conclusión: se recomienda que R/E se pueda usar como un recurso no solo para explicar las repercusiones emocionales de la enfermedad, sino como una estrategia que, de hecho, puede promover una atención más humana e integrada frente a un escenario de pandemia no permanente.

Palabras Clave: Pandemias; Religión; Espiritualidad; Infecciones por Coronavirus.

'Doutor em Psicologia pela Universidade de São Paulo. Docente do Departamento de Enfermagem Psiquiátrica e Ciências Humanas da Escola de Enfermagem de Ribeirão Preto da Universidade de São Paulo (EERP-USP). '2Doutorando em Ciências pelo Programa de Pós-graduação em Enfermagem Psiquiátrica da EERP-USP. ${ }^{3}$ Doutoranda em Ciências pelo Programa de Pós-graduação em Enfermagem Psiquiátrica da EERP-USP. ${ }^{4}$ Doutora em Psicologia pela Universidade de São Paulo. Docente do Centro Universitário das Faculdades Associadas de Ensino (UNIFAE). ${ }^{5}$ Doutora em Psiquiatria e Psicologia Médica pela Universidade Federal de São Paulo. Professora Titular do Departamento de Enfermagem Psiquiátrica e Ciências Humanas da Escola de Enfermagem de Ribeirão Preto da Universidade de São Paulo (EERP-USP).

\section{Como citar este artigo:}

Scorsolini-Comin F, Rossato L, Cunha VF, et al. A Religiosidade/Espiritualidade como Recurso no Enfrentamento da Covid-19. Revista de Enfermagem do Centro Oeste Mineiro. 2020;10:e3723. [Access__]; Available in:___ DOI: http://doi.org/10.19175/recom.v10i0.3723 


\section{INTRODUÇÃO}

A religiosidade/espiritualidade (R/E) vem sendo reconhecidamente evocada na literatura científica como um dos vértices do cuidado em saúde a partir de sua inclusão no conceito multidimensional de saúde da Organização Mundial da Saúde (OMS) em $1998^{(1)}$. Ainda que seja mencionada como dimensão do cuidar e que tenha sido alvo de diversos estudos em diferentes áreas, como a Psicologia, a Medicina e a Enfermagem, o modo como podemos, de fato, incorporar a R/E na linha de atenção em saúde tem sido alvo de amplos debates. Tais reflexões resvalam, quase sempre, em variáveis como os dilemas éticos, a falta de formação de profissionais habilitados para esse cuidado, tanto na graduação como na pós-graduação em saúde, bem como em dificuldades práticas, o que envolve desde justificativas como a falta de tempo como ausências de diretrizes e protocolos capazes de balizar o modo como pode se dar a operacionalização desse vértice na saúde. Ainda que tais dificuldades sejam frequentemente relatadas na literatura científica, permanece $o$ desafio de construção de conhecimentos que possam fazer as práticas em saúde avançarem no que tange ao cotejamento dessa dimensão $0^{(2-5)}$.

Neste estudo, empregamos a terminologia combinada, $R / E$, como forma de incluir as definições de religião, religiosidade e espiritualidade sob uma mesma perspectiva analítica, conforme tem se adensado no campo da saúde ${ }^{(3-4)}$. Ainda que consideremos as diferenças terminológicas e as tradições epistemológicas que envolvem essas definições, para o seu emprego no campo da atenção em saúde, destacamos que o termo combinado parece sumarizar diversos aspectos que poderiam ser desconsiderados caso optássemos por uma ou outra noção em particular. Para os efeitos das recentes investigações da R/E na saúde, esse conceito pode ser definido como o modo como o ser humano se relaciona com uma dimensão externa a ele, que pode fazer referência ou não a instituições e práticas religiosas, recobrindo uma série de fenômenos que coloquem o ser humano em contato com o transcendente. Por essa definição suficientemente ampla podemos incluir, aqui, menções a religiões, práticas, rituais, literaturas religiosas e, até mesmo, apreciações que não envolvam quaisquer símbolos religiosos, mas sim uma perspectiva de contato transcendental ${ }^{(3-4)}$.

Por um longo período, ciência e R/E eram vistas como áreas totalmente distintas e opostas.
Mesmo na atualidade ainda predominam, no âmbito da ciência, concepções racionalistas e mecanicistas que tendem a desvalorizar aquilo que não pode ser totalmente mensurado, previsto ou controlado pela tecnologia e pela razão(6). 0 que se observa é que esses posicionamentos partem da premissa de que não existem comprovações, por meio de aferição, de que a R/E exerça domínio sobre as condições de saúde física das pessoas, ao mesmo tempo em que não se consideram as interferências dela nos aspectos subjetivos e psicológicos dos sujeitos. Consequentemente, as inter-relações entre saúde física, psicológica, aspectos subjetivos, sociais e condições religiosas e espirituais dos indivíduos não são consideradas, demonstrando uma visão fragmentada do ser humano que já deveria ter sido superada ${ }^{(3)}$.

Embora a literatura tenha sido virtuosa em reconhecer e considerar a R/E como vértice de cuidado $^{(1)}$, a cada novo processo de saúde-doença deflagrado é necessário que possamos revisitar os conhecimentos produzidos acerca dessa dimensão ${ }^{(1-6)}$ e como ela pode contribuir para oferecer uma resposta a esse cenário. 0 surgimento do novo coronavírus e da pandemia da COVID-19 tem provocado mudanças nos mais diversos aspectos do cuidado, mobilizando pesquisadores e profissionais em termos dos possíveis tratamentos, da exploração das formas de contágio e de contenção da pandemia, bem como dos aspectos emocionais disparados em função desse importante cenário de saúde global. Como se trata de um contexto recente, as referências acerca da R/E, no contexto da pandemia, ainda estão sendo produzidas, evidenciando-se a importância da presente reflexão no direcionamento dessas propostas. Para tanto, apresentaremos como a pandemia tem se apresentado e quais as principais evidências existentes acerca da saúde mental até o momento, a fim de que tais associações permitam um diálogo com o campo da R/E.

O início da proliferação da COVID-19 se deu de forma rápida e atingiu a maioria dos países do mundo em questão de meses, causando profundos impactos em todos os aspectos da vida cotidiana das pessoas. Em 31 de dezembro de 2019, a OMS foi alertada sobre vários casos de pneumonia na província de Hubei na China ${ }^{(7)}$. Os primeiros casos que apareceram estavam relacionados a um mercado de frutos do mar, na cidade de Wuhan, capital da província central de Hubei, que foi fechado em janeiro de 2020 como 
uma medida de contenção da disseminação da doença $^{(8)}$.

Após o aparecimento dos casos na China, o vírus da COVID-19 se espalhou por diversas regiões do planeta, colocando uma parcela significativa da população mundial em isolamento social preventivo, enquanto uma solução para a doença não é encontrada. Embora muitos detalhes do surgimento desse vírus - como sua origem e sua capacidade de se espalhar entre os seres humanos - permaneçam desconhecidos até o presente momento, um número crescente de casos parece ser resultado da transmissão de humano para humano ${ }^{(9)}$.

A doença, popularmente conhecida como coronavírus (COVID-19), é caracterizada pela síndrome respiratória aguda grave causada pelo coronavírus 2 (SARS-CoV-2). Trata-se de uma emergência de saúde global em andamento, sendo que até 24 de fevereiro de 2020, mais de 80.000 casos da doença haviam sido confirmados, incluindo mais de 2.700 mortes em todo o mundo, afetando pelo menos 37 países $^{(10)}$. Em razão das proporções que assumiu, em 30 de janeiro de 2020, a OMS declarou que o surto da doença constituía-se uma Emergência de Saúde Pública de Importância Internacional - o mais alto nível de alerta da Organização, conforme previsto no Regulamento Sanitário Internacional e, em 11 de março de 2020, declarou-a como uma pandemia $^{(7)}$.

Os dados apresentados na publicação científica referentes aos casos da COVID-19 em fevereiro de 2020 seriam apenas uma ponta do iceberg, evidenciando o que a doença estava causando, o que iria se tornar e o que ainda poderá implicar para a população global se continuar se proliferando. Dados atualizados da OMS, em 31 de março de 2020, apresentam que, no mundo, havia 750.890 casos de COVID-19 (57.610 novos em relação ao dia anterior) e 36.405 mortes (3.301 novas em relação ao dia anterior) até a referida data ${ }^{(7)}$, sendo que, a cada dia, os casos se multiplicam de forma vertiginosa. O Brasil confirmou 5.717 casos e 201 mortes até a tarde do dia 31 de março de 2020. Em 25 de maio, menos de dois meses após o levantamento desses dados em tela, o cenário já apontava para mais de 360 mil casos e 22 mil mortes. Atualmente, em 16 de outubro de 2020, o Brasil contabiliza mais de 5 milhões de pessoas infectadas e 150 mil óbitos. No mundo, esses valores chegam a quase 39 milhões de infectados e mais de um milhão de mortes.
A maioria das pessoas infectadas pela COVID-19 experimentará doença respiratória leve a moderada e se recuperará sem a necessidade de tratamento especial. Os idosos e aqueles com problemas médicos subjacentes, como doenças cardiovasculares, diabetes, doenças respiratórias crônicas e câncer, têm mais probabilidade de desenvolver complicações decorrentes da doença ${ }^{(11)}$ e irem a óbito. Entre os principais sintomas apresentados pelas pessoas com a doença estão: febre, tosse, dores de cabeça, entre outros.

Uma pesquisa realizada em Pequim ${ }^{(12),}$ com 262 pacientes infectados com a COVID-19, apresentou que os sintomas mais comuns do início da doença foram febre $(82,1 \%)$, tosse $(45,8 \%)$ e fadiga $(26,3 \%)$, dispneia $(6,9 \%)$ e dor de cabeça $(6,5 \%)$, casos graves com dispneia $(32,6 \%, 15$ de 46). Outros dados ainda evidenciam que o tempo médio entre o caso sintomático de contato e o início da doença, chamado período de incubação, foi de 6,7 dias, do início da doença ao hospital foi de 4,5 dias, do hospital ao caso confirmado definido foi de 2,1 dias ${ }^{(12)}$.

A cada dia os números aumentam significativamente e, consequentemente, a preocupação social com a doença também, sendo que medidas preventivas mais drásticas têm sido adotadas pelos países, para a contenção da doença, sendo a quarentena uma delas. Consequentemente, diante da apreensão global da pandemia, somada ao distanciamento social e ao isolamento decorrente das medidas de prevenção de transmissão da COVID-19, entre outros problemas, o impacto na saúde mental da população é inevitável e constitui-se como um importante fator que deve ser considerado, pois interferirá na qualidade de vida das pessoas durante e após este período ${ }^{(14-16)}$.

Algumas medidas preventivas podem contribuir para minimizar o volume de contaminações e disseminação do vírus sendo distintas entre $\mathrm{si}^{(13)}$. De acordo com os autores, o distanciamento social tem a finalidade de reduzir as interações das pessoas em uma comunidade mais ampla, antes de se confirmar a infecção e, principalmente, em casos onde a exposição da doença não aconteceu ou é incerta, sendo aplicada, por meio de intervenções como, por exemplo, fechamento de escolas ou escritórios, suspensão de mercados públicos e cancelamento de encontros com várias pessoas ${ }^{(13)}$. $\mathrm{Na}$ quarentena, existe a restrição da circulação de pessoas que foram presumivelmente expostas, 
mas que não estão doentes, seja por não estarem infectados ou por estarem no período de incubação do vírus. Nesse período, todos os indivíduos devem ser monitorados, no caso de ocorrência de qualquer sintoma. Por fim, o isolamento é a medida de afastamento do indivíduo contaminado de quaisquer pessoas não infectadas para protegê-las. Apesar de ser uma medida importante, costuma ser eficaz em interromper a transmissão se a detecção for precoce. Caso contrário, o isolamento costuma ser insuficientemente eficaz para interromper a transmissão e controlar uma pandemia.

Embora não existam dados correlacionando estes eventos, estudo indica que pacientes com confirmação ou suspeita da COVID-19 podem sentir medo das consequências da infecção por um novo vírus, potencialmente fatal, e os que estão em quarentena podem sentir tédio, solidão e raiva. Além disso, o estudo apresenta que sintomas da infecção, como febre, hipóxia e tosse, além de efeitos adversos do tratamento, como insônia causada por corticosteroides, podem levar ao agravamento da ansiedade e do sofrimento mental ${ }^{(14)}$.

A população em geral também sofre os impactos da doença ao ter que lidar com os sentimentos de incerteza, as angústias geradas pelos altos índices de mortalidade da doença, a falta de acesso a recursos básicos para prevenção e a serviços de saúde adequados, o impacto econômico causado por diversos fatores, 0 distanciamento social, entre outros aspectos. Estudo realizado com 1210 entrevistados, com o objetivo de analisar o impacto da COVID-19 na saúde mental das pessoas, evidenciou que do total de entrevistados, 296 (24,5\%) relataram impacto psicológico mínimo, 263 (21,7\%) classificaram impacto psicológico leve e $651(53,8 \%)$ relataram impacto psicológico moderado ou grave ${ }^{(15)}$. Os níveis de depressão, ansiedade e estresse dos entrevistados (avaliados por meio da DASS 21), revelou que, para a subescala de depressão, 843 (69,7\%) foram considerados com pontuação normal, $167(13,8 \%)$ com depressão leve, 148 $(12,2 \%)$ com depressão moderada e 52 (4,3\%) com depressão grave e extremamente grave ${ }^{(15)}$. Em relação à ansiedade, os autores descrevem que $770(63,6 \%)$ foram considerados com escore normal, 91 (7,5\%) avaliados com ansiedade leve, $247(20,4 \%)$ considerados com ansiedade moderada e $102(8,4 \%)$ com ansiedade severa e extremamente severa ${ }^{(15)}$. Quanto ao estresse, o estudo encontrou que 821 (67,9\%) foram considerados com pontuação normal, 292 (24,1\%) avaliados com estresse leve, $66 \quad(5,5 \%)$ considerados com estresse moderado e $31(2,6 \%)$ apresentavam estresse severo e extremamente severo (escore: $27-42)^{(15)}$.

Assim como acontece com a população em geral, os profissionais de saúde também são impactados emocionalmente pelos aspectos da COVID-19, pois estão na linha de frente do combate a essa doença. Um estudo evidenciou que os profissionais de saúde, especialmente aqueles que trabalham em hospitais que cuidam de pessoas com pneumonia confirmada ou suspeita de COVID-19, são vulneráveis a alto risco de infecção e problemas de saúde mental. Neste estudo, também foi apresentado que os profissionais de saúde podem ter medo de contágio e espalhar o vírus para suas famílias, amigos ou colegas ${ }^{(14)}$.

Assim, percebe-se que a severa situação vivenciada pela pandemia da COVID-19 pode ser um agravante para as condições de saúde mental dos profissionais de saúde que estão lidando com a doença. Estudo aponta que a situação vivenciada no trabalho de tratamento da doença propiciou o aparecimento de problemas de saúde mental, como estresse, ansiedade, sintomas depressivos, insônia, negação, raiva e medo. Segundo seus autores, esses problemas tornam-se complexos, pois afetam a atenção, o entendimento e a capacidade de tomada de decisões dos trabalhadores, o que pode dificultar a luta contra a COVID-19, além de ter um efeito duradouro no bem-estar geral ${ }^{(16)}$.

Considerando que a R/E pode funcionar como uma estratégia de enfrentamento diante de eventos estressores e com forte mobilização emocional( $^{(2-6)}$, como podemos observar a partir dos primeiros levantamentos em saúde mental a partir da pandemia da COVID-19 ${ }^{(14-15)}$, aventa-se que esse recurso possa ser empregado nesse contexto, fornecendo importantes direcionadores para pacientes, familiares e também profissionais de saúde. Também considerando a emergência da temática da morte, nesse contexto, conjectura-se que a R/E possa fornecer subsídios para a elaboração de protocolos de cuidado que, de fato, considerem essa dimensão na atenção à saúde, retomando as recomendações da $\mathrm{OMS}^{(1)}$.

Nota-se que a COVID-19 tem representado uma pandemia com importantes repercussões em todos os setores da atividade humana, despertando o interesse e a necessidade de produção de evidências que possam, em conjunto, 
oferecer uma resposta a esse fenômeno e suas associações com demais variáveis. Em que pesem os recursos já desenvolvidos e em discussão nesse cenário, bem como forma de contribuir com a produção de conhecimentos e reflexões acerca desse evento de proporções globais, este estudo se aterá à dimensão da R/E.

A escolha da R/E retoma não apenas as suas associações com os desfechos positivos em saúde $^{(2,6)}$, como também a necessidade de discutir esse tema em um contexto pandêmico e ainda considerado novo tanto por pesquisadores da área de saúde mental ${ }^{(14-15)}$ quanto da R/E. Em um cenário impermanente e com diversas pesquisas em curso, ainda não foram encontrados estudos empíricos que forneçam evidências acerca da $R / E$, nesse contexto, nem em termos da avaliação da R/E de pessoas adoecidas, de familiares e de profissionais de saúde, nem em termos da utilização da $R / E$ como estratégia de enfrentamento, nesse cenário de forte mobilização emocional. Aventa-se que cenário abarque a necessidade de discussão acerca da morte e do morrer, o que tem sido frequentemente apresentado como um elemento capaz de evocar uma escuta voltada à R/E por parte de diferentes profissionais ${ }^{(3-4)}$. A partir desse panorama e da lacuna de produção identificada em um contexto ainda recente, objetivou-se, nesta reflexão teórica, problematizar de que modo a $\mathrm{R} / \mathrm{E}$ pode ser empregada como um recurso no enfrentamento da pandemia da COVID-19.

\section{MÉTODO}

Trata-se de uma reflexão teórica amparada na literatura científica produzida acerca da $R / E^{(1-6)}$, articulando como essa dimensão pode auxiliar o enfrentamento de situações de adoecimento. Os estudos de reflexão teórica não podem ser considerados estudos de revisão, haja vista que o cotejamento de evidências, nessas produções, é substituído por um denso processo analítico que se baseia em diversos elementos presentes na literatura, envolvendo a incorporação de diferentes níveis de evidência e de diferentes indícios para a discussão. Em um contexto ainda incipiente em relação à produção de conhecimentos capazes de fornecer evidências robustas para a prática em saúde, como em relação à pandemia da COVID-19, tal reflexão teórica mostra-se oportuna por permitir aproximações capazes de produzir não evidências, mas sim indícios capazes de contribuir para o delineamento de futuros estudos empíricos e demais percursos analíticos derivados de um percurso ainda exploratório. Assim, o que legitima esse tipo de estudo é justamente o vigor reflexivo que pode ser disparador de importantes convites ao conhecimento, sobretudo no campo das ciências da saúde ${ }^{(17)}$.

Para a discussão proposta também serão empregados estudos disponíveis sobre a pandemia de COVID-19 ${ }^{(14-15)}$ para que se possam delimitar as recomendações de como empregar a R/E como recurso no cuidado em saúde. Como apresentado na introdução, como ainda são incipientes os estudos empíricos acerca da R/E no contexto da pandemia, recorrer-se-á à produção consolidada sobre a $R / E$ e seu emprego em cenários de cuidado à saúde ${ }^{(1-6)}$, a fim de que tais conhecimentos possam subsidiar as discussões aqui tecidas, tendo como norte os desafios do cuidado em meio à pandemia de uma doença ainda bastante desconhecida(14-16).

Para a organização dessa reflexão teórica foram produzidas cinco categorias que revelam possibilidades de compreensão do fenômeno em tela - aplicação da R/E no contexto da pandemia da COVID-19 - e que representam, a partir dos estudos aqui reunidos e das investigações correntes no contexto da R/E, uma resposta aos desafios apresentados na justificativa do estudo e que atravessam diretamente a elaboração de tais recomendações. Como ainda não há elementos para a construção de uma revisão sistemática, tais categorias foram produzidas tendo como norte a literatura consolidada acerca da R/E e seu emprego no contexto da saúde ${ }^{(2-3,5-6,18-23)}$, representando vértices reflexivos, como o conceito de coping religioso-espiritual ${ }^{(18)}$, por exemplo, que podem nortear a aproximação da dimensão da R/E com o contexto pandêmico contemporâneo, haja vista as primeiras evidências disponíveis ${ }^{(13-16)}$. Desse modo, tais categorias foram propostas como forma de articular a literatura da R/E em saúde com os possíveis pontos de encontro a partir dos conhecimentos disponíveis acerca da pandemia da COVID-19, permitindo inteligibilidades em um contexto ainda exploratório.

\section{RESULTADOS E DISCUSSÃO}

\section{$R / E$ como recurso para compreensão dos efeitos da pandemia na vida cotidiana}

Nesta reflexão, aventa-se que a R/E pode ser empregada como um recurso, em níveis individual e coletivo, para compreender ou enfrentar os efeitos adversos decorrentes da 
pandemia que têm afetado a vida cotidiana em escala global. Entre os efeitos que podem ser assinalados - já descritos ou ainda por emergir -, destacamos: (a) repercussões emocionais em função das restrições sociais tanto como medida de saúde pública para o retardamento do contágio comunitário e do isolamento ou quarentena, no caso de pessoas infectadas ou que tiveram algum nível de exposição ao vírus; (b) mudanças sociais, culturais e familiares em função da morte e do adoecimento de pessoas próximas, deflagrando a necessidade de reestruturação de posicionamentos, de desempenho de papéis e de funções desenvolvimentais, por exemplo, no núcleo familiar; (c) construção de um sentimento coletivo de pertencimento e de responsabilidade com o humano, tornando premente a emergência da empatia como forma de aproximar pessoas que têm passado por situações semelhantes e evocando a consolidação de redes de apoio social que ultrapassem estruturas familiares ou próximas em termos contextuais e ambientais; (d) necessidade de adaptação nos mais diversos cenários da vida, como em relação às escolas, universidades e equipamentos de saúde.

Há que se destacar que essa produção encontra assentamento no epicentro de uma crise de saúde, o que, obviamente, pode disparar mudanças em curto, médio e longo prazos ${ }^{(8-13)}$ com que esses efeitos são aqui descritos e problematizados. No que tange especificamente à $\mathrm{R} / \mathrm{E}$ como elemento ligado ao transcendente ${ }^{(5)}$, percebe-se que tais efeitos - e diversos outros que emergirão nos próximos tempos - poderão ser compreendidos tendo como norteador o modo como o sujeito está ligado àquilo que não necessariamente está presente em sua materialidade ${ }^{(2-3)}$.

A sua conexão com o transcendente e/ou com o divino pode funcionar como mecanismo capaz de manter a capacidade crítica, de apoio na realidade e na tomada de decisões da vida cotidiana que, de outra forma, estariam em suspenso $^{(4-6)}$. A R/E oferece ao sujeito uma possibilidade de conexão e de descrição de si para além dos critérios materiais, pode ser um recurso de manutenção de saúde, de construção de outras estratégias que deem sentido às vivências, por vezes dolorosas, decorrentes de qualquer pandemia. Esse transcendente e/ou divino pode ser exemplificado por meio do contato com santos, entidades, espíritos, livros sagrados, participação em rituais religiosos e em experiências corporais que promovam essa conexão, perseveração em princípios religiosos caros ao sujeito, contato com a natureza, abertura a experiências sensoriais, entre outros ${ }^{(4,6)}$.

Assim, a R/E seria alçada a uma condição que permitiria ao sujeito uma vivência menos dissociada de seu contexto e, por isso mesmo, ampliando as possibilidades de resposta às adaptações necessárias ${ }^{(1-3)}$. Nesse ponto, cabe ressaltar que também se compreendem os efeitos negativos da R/E na vida cotidiana, em situações em que esta pode ser um mecanismo mobilizador de angústias espirituais e desresponsabilização frente a situações que exigiriam respostas contundentes ${ }^{(18)}$ ou mesmo quando mobiliza as pessoas a manifestação de atitudes fanáticas e radicais que possam ser prejudiciais ${ }^{(19)}$. Também cabe ressaltar que se compreendem as controvérsias, polêmicas e conflitos éticos envolvendo questões ligadas às crenças religiosas/espirituais e os cuidados em saúde como expresso em publicação científica ${ }^{(20)}$. Desse modo, o enfoque dado não desconsidera os aspectos negativos da $R / E$, porém, parte do princípio que esta deve ser considerada como uma dimensão humana que é valorizada pela sociedade e, principalmente, nos contextos de atenção à saúde ${ }^{(1)}$. Nessa perspectiva, permite-se analisar e compreender a R/E em uma percepção mais amadurecida da realidade, o que, de fato, pode promover a adoção de estratégias e ações mais propositivas.

\section{R/E como recurso de enfrentamento e fonte de apoio para pessoas adoecidas, cuidadores e familiares}

Sabe-se que a R/E se constitui como uma dimensão que perpassa a vida humana desde os primórdios da civilização, sendo um importante elemento sociocultural que interfere nos modos de vida, nas condições de saúde e nos aspectos subjetivos das pessoas ${ }^{(1-2)}$. A interlocução entre $\mathrm{R} / \mathrm{E}$ e cuidado em saúde ainda encontra resistências na atualidade, principalmente pelo fato de em inúmeras ocasiões substituir-se o tratamento convencional pelo tratamento religioso/espiritual, o que não seria indicado, pois pode comprometer ainda mais as condições de saúde da pessoa adoecida ${ }^{(5)}$. Além disso, a postura de determinados líderes religiosos e espirituais, em muitas ocasiões, oportuniza condições para que as pessoas deixem de ter comportamentos adequados de saúde, sejam criticadas em relação ao que vivem e acabem seguindo orientações que não são adequadas e sejam orientadas em 
interpretações e concepções pessoais do líder religioso/espiritual, sem embasamento concreto sobre o assunto ou de outras ordens que compõem o sujeito.

A R/E constitui-se como dimensão que atua nos aspectos subjetivos das pessoas, oportunizando conforto e amparo diante de situações difíceis, cria condições para o enfrentamento de momentos de crise e auxilia na elaboração de aspectos que são complexos de serem compreendidos e solucionados de forma concreta $^{(4-5)}$. Desse modo, ela deve ser considerada enquanto elemento ligado diretamente aos aspectos psicológicos/subjetivos dos sujeitos. Utilizá-la enquanto uma ferramenta para direcionar posicionamentos e comportamentos prejudiciais à própria saúde ou de outrem é minimamente questionável e deve ser problematizado.

Pesquisas recentes apontam a R/E como um dos aspectos mais significativos da subjetividade humana, observando-se que se relaciona com a construção de sentido e ordenação de vida dos indivíduos, influenciando também a sua saúde de forma positiva ${ }^{(21)}$. Em situações de adversidade a $R / E$, quando utilizada de forma prudente, deixando de lado os aspectos ligados ao fanatismo religioso e espiritual, pode ser importante aliada no processo de enfrentamento ou coping religioso-espiritual(18). É comum as pessoas se voltarem para a $\mathrm{R} / \mathrm{E}$ em tempos de adversidade como uma maneira de entender aquilo que vivem e buscar condições e respostas para reagirem diante das situações a que são expostas ${ }^{(4)}$.

$A R / E$ remete a pessoa para a relação com o transcendente na busca de significados da vida ${ }^{(22)}$. Torna-se uma forma de expressão da identidade e do propósito existencial de cada um mediante a própria história, experiências e aspirações, sendo que o apoio em Deus ou o que considera sagrado ocorre pela necessidade de auxílio religioso/espiritual para melhor enfrentamento do medo, da solidão e do inesperado ${ }^{(23)}$.

Em situações de adoecimento em saúde com doenças que ainda não possuem bons resultados no tratamento ou que tenham um significado social associado ao perigo e à morte ${ }^{(22-}$ ${ }^{23)}$, as pessoas podem recorrer a R/E na tentativa de encontrar suporte para lidar com os aspectos negativos atrelados a esses contextos. Essa busca pelo apoio na espiritualidade ou na religião é conhecida como coping religioso/espiritual $(\mathrm{CRE})^{(18)}$.
O CRE pode ser utilizado positivamente quando faz uso das crenças e comportamentos religiosos/espirituais para a resolução de problemas, prevenção ou alívio das consequências negativas das situações estressantes vivenciadas no momento ${ }^{(18)}$. Nesse sentido, os autores exemplificam que o CRE positivo é o que contempla estratégias com efeito benéfico ao sujeito como, por exemplo, a procura pelo amor/proteção de Deus ou a maior conexão com forças transcendentais. Em contrapartida, o CRE negativo também pode acontecer, o que seria a utilização das crenças e comportamentos religiosos/espirituais que geram consequências prejudiciais como, por exemplo, delegar a Deus a resolução do problema sem qualquer ação por parte do sujeito ${ }^{(18)}$. Com a pandemia, é premente a necessidade de produção de evidências acerca do CRE nesse contexto, possibilitando o desenvolvimento de elementos e práticas que possibilitem a valência positiva desse recurso tanto para pacientes, familiares como para profissionais de saúde.

É importante sempre recuperar que a fé em Deus, no divino e no sagrado é um sentimento arraigado na cultura e tão necessária quanto outros modos de enfrentamento ${ }^{(1)}$, pois a dimensão espiritual ocupa um lugar de destaque na vida das pessoas, sendo imprescindível que se conheça a R/E dos usuários para o planejamento do cuidado $^{(23)}$. A R/E faz parte da construção da personalidade de cada ser humano, uma expressão da identidade e propósito, à luz da sua história, experiência e aspiração, produz alívio ao sofrimento, na medida em que permite mudança na perspectiva subjetiva pela qual o paciente e a comunidade percebem o contexto da doença ${ }^{(23)}$.

\section{R/E como suporte nas situações de quarentena, distanciamento e isolamento social}

Como ainda não há protocolos que apontem para a efetividade de medicamentos e as pesquisas para a produção de vacina estão em curso, as medidas relacionadas à quarentena e ao isolamento e distanciamento social têm sido adotadas e recomendadas por diferentes governos como forma de achatamento nas curvas de contágio e, consequentemente, de diminuição da sobrecarga dos equipamentos de saúde ${ }^{(13)}$.

Em todos esses casos, é imposto aos sujeitos um cerceamento, com maior ou menor grau, para uma nova situação que implicará em mudanças significativas na sua rotina, assim como limitações de atividades que ocasionarão perdas 
diversas. Essas situações apresentadas podem ser aproximadas ao que é conhecido como desamparo aprendido. O desamparo aprendido vem sendo entendido como uma resposta do sujeito a uma redução da responsividade do organismo ao ambiente, o qual tem uma experiência de incontrolabilidade, associando-se ao fenômeno da depressão(24). Apesar de ser um modelo experimental, ele pode ser transposto para uma interpretação clínica, levando a acreditar que as situações de restrição social empreendidas colocam em risco aspectos psicológicos que, se não devidamente cuidados, podem gerar outros tipos de acometimentos que também sobrecarregarão o sistema de saúde, por exemplo, em termos de saúde mental ${ }^{(14-16)}$.

Outra preocupação do impacto que a restrição/isolamento social pode ocasionar é aumentar o sentimento de solidão. Sobre esse ponto, estudos apontam que existem diferentes impactos do isolamento social e solidão na saúde e que, além de maior chance de depressão, existe um maior comprometimento cardiovascular e aumento da mortalidade por todas as causas ${ }^{(24)}$. Nos idosos, essas repercussões já existiam antes mesmo da pandemia e são particularmente problemáticas, já que são um público que tem seus recursos econômicos e sociais rebaixados pela interrupção da atividade profissional, de limitações funcionais, da morte de parentes e cônjuges e mudanças nas estruturas família e mobilidade ${ }^{(24)}$. Para evitar o agravamento dessas situações faz-se importante o incentivo redobrado de estratégias de prevenção e minimização desses acometimentos.

Ao restringir a transição e frequência aos ambientes coletivos de congregação religiosa/espiritual, limita-se o sujeito de praticar a sua $R / E$ organizacional, voltada para comportamentos ligados ao contexto da instituição religiosa/espiritual, assim como ao desempenho de funções e cargos que pode ocupar em uma congregação. Nesse aspecto, evidências apontam que essa assiduidade tem um impacto positivo para a redução do nível de mortes, em baixos níveis de depressão e melhores níveis de saúde, mas que entre os idosos essa frequência é naturalmente reduzida por outras limitações de saúde desse público, sugerindo que eles enfatizem e se engajem mais em sua R/E intrínseca e não organizacional ${ }^{(24)}$. Acredita-se que diante das medidas de restrições sociais que vêm sendo aplicadas em resposta à pandemia, todos, idosos ou não, podem fazer uso mais enfático de vivências religiosas/espirituais não organizacionais e intrínsecas. Ou que a própria R/E organizacional possa ser vivenciada a partir de novas maneiras de estar e se fazer presente, a partir das tecnologias, por exemplo.

$\mathrm{Na}$ R/E vivenciada fora do setting de uma determinada religião/instituição, enfatiza-se a busca pela crença e comportamentos religiosos/ espirituais sem lugar ou tempo específico, sem qualquer forma preestabelecida, que pode ser evocada nos momentos em que se instalam dúvidas ou desesperança para a superação da dificuldade. Estratégias como preces e orações, conversar com Deus ou com o que se tem por divino, leituras de escritos religiosos/espirituais, rituais privados, meditação, mídias religiosas/espirituais podem ser vivenciadas nesse momento de restrição social. Já a R/E intrínseca compõe a dimensão subjetiva de quanto e como o sujeito percebe a R/E em sua vida, em que se busca a internalização e vivência plena da $\mathrm{R} / \mathrm{E}^{(3,5,18)}$. Como parâmetros dessa relação intrínseca têm-se o sentir a presença de Deus ou conectado com o divino e/ou sagrado (que seria oposto ao sentimento de solidão), condutas e comportamentos que vão ao encontro e de acordo com as crenças pessoais e que norteiam a maneira de viver ${ }^{(3)}$.

A presença e vivência proporcionada pelas três possíveis relações do sujeito com a R/E sugerem, a partir de outros estudos que não ligados diretamente à COVID-19, que existe uma relação positiva da R/E com o estilo de vida, a qualidade de vida, felicidade e promoção de saúde ${ }^{(3,6)}$, sendo um recurso disponível, acessível e que pode ser investido e incentivado nesse momento crítico vivenciado mundialmente.

\section{R/E como recurso para profissionais de saúde} diretamente envolvidos no combate à pandemia A situação pandêmica tem proporcionado um contato cada vez mais direto entre a população e os equipamentos de saúde. Mesmo em situações de parco acesso à saúde, a população atingida tem sido recebida nos equipamentos de saúde para o tratamento de COVID-19. Nesse sentido, embora diferentes sistemas de saúde possam existir, a exemplos dos informais e dos culturais (folk), o sistema formal tem sido elencado como o mais adaptado nesse cenário em que tratamentos e condutas cientificamente embasadas podem oferecer respostas mais efetivas. 
Nessa maior aproximação entre pacientes e profissionais de saúde, pelo fluxo cada vez maior de frequência de pessoas infectadas aos hospitais e centros de referência no tratamento da COVID19, um efeito é o de que o espaço do cuidado formal passa a ser um ambiente desenvolvimental, ou seja, passa a ser um contexto no qual há o desenvolvimento do sujeito. Isso permite que mais pessoas possam ser cuidadas dentro do sistema formal, o que aumentam as chances de uma resposta mais exitosa em termos de cura e de restabelecimento da saúde.

No entanto, um aspecto que deve ser discutido é o de que todo o contato corporal entre equipe de saúde e paciente acabe sendo fragilizado, sendo mediado por equipamentos de proteção indispensáveis e que acabam, de algum modo, afastando esses sujeitos em interação. 0 contato não apenas mediado por esses equipamentos, mas também o medo desses profissionais se infectarem, no momento de consultas e de tratamentos, podem comprometer a própria assistência em saúde, gerando afastamento, estigma e falta de acolhimento. Em que pesem as regras e protocolos de proteção aos pacientes e equipes de saúde, essas características do atendimento em um contexto de pandemia podem ser consideradas, em uma leitura mais estreita, como desumanizadoras. Assim, a R/E pode ser um aspecto a ser trabalhado pelas equipes e com os próprios pacientes ${ }^{(4,16)}$, podendo funcionar como uma dimensão sobre a qual podemos refletir em busca de inteligibilidades, em busca de um acolhimento que não pode ser oferecido, por exemplo, a partir do toque e de uma proximidade corporal maior.

A R/E pode ser um elemento que contribua decisivamente na humanização do cuidado, uma vez que os profissionais de saúde, cada vez mais, estarão em contato com essa dimensão na atenção aos seus pacientes. Se a R/E é um elemento mencionado na emergência de situações de maior mobilização emocional ou, então, quando a morte torna-se um evento mais próximo, a exemplo dos cuidados paliativos ${ }^{(4)}$, aventa-se que a pandemia de COVID-19, pelos seus diversos efeitos, possa aproximar os profissionais de saúde, de diferentes linhas do cuidado, com esses elementos. Assim, a R/E tanto seria uma dimensão mais evocada na atenção à saúde como uma possível repercussão da pandemia na vida coletiva como também estaria mais presente no cotidiano profissional justamente por esse dia a dia estar cada vez mais permeado pelas questões acerca da morte e do morrer, assim como discutido na próxima categoria.

Um último sentido que pode ser mencionado, nessa categoria, refere-se justamente à possibilidade desse profissional de saúde que tem atuado no combate à pandemia entrar em contato com a própria R/E. Em um cenário de atenção emergencial e de proporções que fogem ao que é esperado ou à experiência cotidiana, na maioria dos equipamentos de saúde, esses profissionais podem se beneficiar de um maior contato com o transcendente por diversos motivos $^{(4)}$ : (a) possibilidade de maior compreensão do cenário atual e da importância do papel dos profissionais de saúde na tentativa de fazer frente à pandemia; (b) possibilidade de vivenciar na $\mathrm{R} / \mathrm{E}$ uma compreensão acerca do próprio papel profissional( ${ }^{(4)}$, manifestando empatia pelos pacientes e pessoas que recorrem a esses serviços; (c) possibilidade de empregar os conhecimentos da R/E para melhor contato com os pacientes em um contexto de maior mobilização emocional ${ }^{(5)}$; (d) emprego da R/E para lidar com a maior exposição à morte de pacientes; (e) emprego da R/E para lidar com a maior frequência de situações nas quais o manejo dos profissionais de saúde possa não promover qualquer alívio ou que a atuação profissional esbarre em condições irreversíveis, abrindo a possibilidade de maior contato com situações inesperadas e nas quais os conhecimentos profissionais não dão conta de oferecer respostas.

Essas condições podem tornar o profissional de saúde mais poroso não apenas ao emprego da R/E no cuidado em saúde, como à utilização da sua própria R/E em seu autocuidado, na tentativa de criar inteligibilidades em um cenário pandêmico em que muitas respostas se mostram em aberto. Postula-se que o contato com a própria R/E possa tornar esses profissionais mais "próximos", experiencialmente de seus pacientes, o que pode ter um efeito positivo quando analisamos a oferta do cuidado(4). Em um contexto em que muitos conhecimentos estão em desenvolvimento e para o qual os protocolos são criados e recriados em uma velocidade cada vez maior, a $R / E$ parece ser um vértice perene para a maior humanização do cuidado ${ }^{(1-2,4)}$, pois se trata de uma dimensão que, independentemente das condições, continua em seu papel de produzir respostas com maior capacidade de encontro com o sujeito e as suas diferentes necessidades. 


\section{R/E como recurso na compreensão de situações de luto}

Como se trata de uma doença com alta mortalidade, sobretudo na população com mais de 60 anos de idade e com comorbidades, como as doenças crônicas, as discussões acerca dos processos de morte, morrer e de enlutamento fazem-se cada vez mais prementes. O processo de luto tem sido alvo de reflexões de filósofos, psicólogos e de diferentes profissionais de saúde ao longo da história, cada vez mais interessados no modo como esse evento pode ser tanto uma resposta a um sofrimento extremo como também um disparador da manutenção desse sofrimento, podendo evoluir para uma condição patológica(25). Assim, emergem sentidos que tanto ligam o luto a uma resposta do organismo diante de uma perda como um elemento que pode acompanhar o enlutado, acarretando-lhe diversos prejuízos à adaptação a um novo contexto, em que as perdas e as mortes sejam, de algum modo, inevitáveis, o que se aplica à condição de pandemia.

Entre as estratégias de elaboração do luto encontramos a $R / E$, que pode ser uma resposta ao sofrimento, tanto oferecendo explicações sobre esse fenômeno, como abrindo a oportunidade de que tal vivência, sendo explicada dentro de uma inteligibilidade mais porosa ao sujeito, possa promover conforto e acolhimento diante da impossibilidade de reversão desse quadro. Embora o conceito de R/E não envolva, necessariamente, uma resposta, mostra-se um mecanismo importante para oportunizar ao sujeito uma explicação, uma possibilidade compreensiva ${ }^{(3,25)}$. Desse modo, a R/E pode amparar o sofrimento justamente por partir de explicações que sejam mais palatáveis ao mundo experiencial das pessoas ${ }^{(5,22)}$. Quando se tornam compreendidas nesse sofrimento podem, de fato, lidar de modo mais amadurecido com esse evento.

Diante do cenário atual, o contato com a morte passa a ser cotidiano, não apenas próximo a cada pessoa, mas como um evento global, marcado em notícias, nas diferentes mídias e em todo um repertório de informações produzidos diariamente sobre a COVID-19. Em que pesem as notícias que atualizem a todo dia os números de novos infectados e de mortes em todo o mundo, pode-se dizer que o contato com a dimensão do morrer tornou-se algo cotidiano, o que pode ser apreendido de diferentes maneiras.

Uma delas refere-se à automatização e à naturalização dos processos relacionados à morte e ao morrer ${ }^{(25)}$. Decorrente dessa consideração, o enlutamento ou o estado de luto passa a ser uma experiência não mais individual, mas também coletiva, que atravessa a comunidade. Embora cada pessoa possa desenvolver um modo particular de lidar com esse luto, a depender de suas experiências pregressas, do ente perdido e das condições dessa perda, entre outros fatores, o luto tem passado a ser uma experiência comum, compartilhada por diversas pessoas, em diferentes países.

Ainda que a morte nunca tenha sido uma experiência isolada e apartada do humano, na contemporaneidade tem emergido uma noção que pode aqui ser inaugurada, que é a da onipresença da morte. As investigações sobre a COVID-19, certamente, levantarão o estigma relacionado à morte que emerge como uma condição, ao passo que o luto também passa a ser, também, um elemento coletivo, promovendo, por extensão, o compartilhamento de um sintoma.

É nesse momento de maior desamparo de busca por explicações e formas de aplacar o sofrimento que a R/E pode ser empregada. Essa utilização, no entanto, não deve ter como objetivo um mascaramento da realidade, mas justamente levar o indivíduo e a coletividade à uma real consideração da R/E nas experiências do viver e do morrer. Para além dos clássicos empregos da R/E, sobretudo em momentos de crise e de maior desesperança, como em adoecimentos graves e nos cuidados paliativos, tão bem retratados na produção científica da Enfermagem ${ }^{(22-23)}$, abre-se a possibilidade de uma leitura mais natural da $R / E$, como se o sentido dessa conexão com $O$ transcendente pudesse ser algo que realmente nos constitui.

A partir das reflexões aqui endereçadas, postula-se que a R/E não teria exclusivamente a acepção de recurso, como desenvolvido largamente, neste estudo, e consolidado na literatura $^{(1,18)}$, mas como dimensão de pertencimento ao humano. Corporificando a R/E como realidade e não necessariamente como recurso, poderemos, de fato, nos permitir uma experiência mais integradora e menos ansiosa por respostas, ferramentas e modos de diminuir ou mascarar um sofrimento inevitável, sobretudo em um cenário pandêmico que tem provocado diversas reflexões que permanecem em aberto.

\section{CONSIDERAÇÕES FINAIS}

A partir dos aspectos elencados na presente reflexão teórica, recomenda-se que a R/E possa 
ser empregada não apenas na explicação das repercussões emocionais da doença, mas como estratégia que possa, de fato, promover um cuidado mais humano e integrado diante de um cenário pandêmico impermanente. Para tanto, deve ser considerada tanto como estratégia de enfrentamento quanto como elemento de realidade. Assumindo essa realidade pode-se, com maior propriedade, abrir-se a leituras que não concebam a R/E como algo externo ao sujeito, mas fundamentalmente integrado à experiência humana. Os efeitos da pandemia ainda são pouco conhecidos, de modo que diversos conhecimentos estão sendo produzidos para oferecer mais respostas a um cenário de incertezas que caracteriza a contemporaneidade diante desse grave evento em saúde, com inequívocas repercussões em todas as dimensões da vida humana.

Assim, nesse cenário impermanente, de incertezas e de muitos questionamentos em aberto, a $R / E$ pode ser uma estratégia não que ofereça necessariamente uma resposta, mas que possa abrir a possibilidade de oferta de um lugar de conforto diante do que ainda não se sabe e de um porvir que se apresenta a cada dia envolto em novas problematizações. Diante do que não se sabe, do que não se vislumbra e do muito que pode ocorrer, a R/E parece ser uma dimensão que coteja esse não-saber justamente por oferecer um lugar para que essas reflexões sejam acolhidas e endereçadas. Cuidar desse espaço pode ser uma estratégia potente e humanizadora, religando pessoas, contextos e processos em um mesmo circunscritor desenvolvimental.

\section{REFERÊNCIAS}

1- World Health Organization (WHO). Division of mental health and prevention of substance abuse. WHOQOL and spirituality, religiousness and personal beliefs (SRPB). Genève: World Health Organization; 1998.

2- Moreira-Almeida A, Van Rensburg BJ, Sharma A, Verhagen PJ, Cook $\mathrm{CCH}$. WPA position statement on spirituality and religion in psychiatry. World Psychiatry 2016;15(1):87-8. DOI: 10.1002/wps.20304

3- Koenig HG. Religião, espiritualidade e psiquiatria: Uma nova era na atenção à saúde mental. Rev Psiquiatr Clín. 2007;34(supl 1):5-7. DOI: 10.1590/S0101-60832007000700002

4- Cunha VF, Scorsolini-Comin F. Best professional practices when approaching religiosity/spirituality in psychotherapy in Brazil. Couns Psychother Res. 2019;19(4):523-32. DOI: 10.1002/capr.12241

5- Espíndula JA, Valle ERM, Bello AA. Religião e espiritualidade: Um olhar de profissionais de saúde. Rev Latino-Am Enfermagem 2010;18(6): 1229-36. DOI: $10.1590 /$ S010411692010000600025

6- Melo CF, et al. Correlação entre religiosidade, espiritualidade e qualidade de vida: uma revisão de literatura. Estud Pesqui Psicol. 2015 [citado em 15 jan 2017]; 15(2): 447-64. Available in: http://pepsic.bvsalud.org/scielo.php?script=sci_a rttext\&pid=S1808-42812015000200002\&lng=pt\& $\mathrm{nrm}=$ iso

7- Organização Panamericana de Saúde. Folha informativa - COVID-19 (Doença causada pelo novo coronavírus). Brasília, DF: OPAS, 2020.

8- Mahase E. China coronavirus: What do we know so far? BMJ 2020;368:308. DOI: 10.1136/bmj.m308

9- Munster VJ, Coopmans $M$, et al. A novel coronavirus emerging in China-key questions for impact assessment. N Engl J Med. 2020; 382(8):692-94. DOI: 10.1056/NEJMp2000929

10- Yuen K-S, Ye Z-W, Funge S-Y, Chan C-P, Jin DY. SARS-CoV-2 and COVID-19: The most important research questions. Cell Biosci. 2020;10(1):1-5. DOI: 10.1186/s13578-020-004044

11- World Health Organization (WHO). Coronavirus. Genève: WHO; 2020.

12- Tian S, Hu N, Chen K, Kang X, Xiang Z, Chen H, et al. Characteristics of COVID-19 infection in Beijing. J Infect. 2020;80(4): 401-06. DOI: 10.1016/j.jinf.2020.02.018

13- Wilder-Smith A, Freedman DO. Isolation, quarantine, social distancing and community containment: Pivotal role for old-style public health measures in the novel coronavirus (2019nCoV) outbreak. J Travel Med. 2020;27(2):1-4. DOI: $10.1093 / \mathrm{jtm} /$ taaa020

14- Xiang Y-T, Yang Y, Li W, Zhang L, Zhang $Q$, Cheung $T$, et al. Timely mental health care for the 2019 novel coronavirus outbreak is urgently needed. Lancet 2020;7(3):228-9. DOI: 10.1016/S2215-0366(20)30046-8

15- Wang C, Pan R, Wan X, Tan Y, Xu L, Ho CS, et al. Immediate psychological responses and associated factors during the initial stage of the 2019 coronavirus disease (COVID-19) epidemic among the general population in china. Int J Environ Res Public Health. 2020;17(5):1729. DOI: 10.3390/ijerph17051729 
16- Kang L, Hu S, Chen M, Yang C, Yang BX, Wang $Y$, et al. The mental health of medical workers in Wuhan, China dealing with the 2019 novel coronavirus. Lancet 2020; 7(3):e14. DOI: 10.1016/S2215-0366(20)30047-X

17- Scorsolini-Comin F. Guia de orientação para a iniciação científica. São Paulo: Atlas; 2013.

18- Silva GCN, Reis DC, Miranda TPS, Melo RNR, Coutinho MAP, Paschoal CS, et al. Coping religioso/espiritual e a angústia espiritual em pessoas com câncer. Rev Bras Enferm. 2019;72(6):1534-40. DOI: 10.1590/0034-71672018-0585

19- Akinola O. Boko Haram insurgency in Nigeria: Between Islamic fundamentalism, politics, and poverty. African Security 2015;8(1):1-29. DOI: 10.1080/19392206.2015.998539

20- Tomkins A, Duff J, Fitzgibbon A, Karam A, Mill EJ, Munnings $K$, et al. Controversies in faith and health care. Lancet 2015;386(10005):1776-85. DOI: 10.1016/S0140-6736(15)60252-5

21- Melo CF, Sampaio IS, Souza DLA, Pinto NL. Correlação entre religiosidade, espiritualidade e qualidade de vida: Uma revisão de literatura. Estud Pesqui Psicol. 2015 [citado em 15 jan 2017]; 15(2):447-64. Available in: http://pepsic.bvsalud.org/scielo.php?script=sci_a rttext\&pid=S1808-42812015000200002\&lng=pt\& tlng=pt

22- Gomes MV, Xavier ASG, Carvalho ESS, Cordeiro SC, Ferreira SL, Morbeck AD. "À espera de um milagre": Espiritualidade/religiosidade no enfrentamento da doença falciforme. Rev Bras Enferm. 2019;72(6):1554-61. DOI: 10.1590/00347167-2018-0635

23- Guerrero GP, Zago MMF, Sawada NO, Pinto MH. Relação entre espiritualidade e câncer: Perspectiva do paciente. Rev Bras Enferm. 2011;64(1):53-9. DOI: 10.1590/S003471672011000100008

24- Courtin E, Knapp M. Social isolation, loneliness and health in old age: A scoping review. Health Soc Care 2017;25(3):799-812. DOI: https://doi.org/10.1111/hsc.12311

25- Bowlby J. Perda: Tristeza e depressão. 3a ed. São Paulo: Martins Fontes; 2004.

Nota: Os autores agradecem à CAPES pela bolsa de doutorado concedida aos segundo e terceiros autores, bem como ao CNPq pela bolsa de produtividade em pesquisa concedida ao primeiro e à última autora.

Recebido em: 07/03/20

Aprovado em: 26/05/20
Endereço de Correspondência: Departamento de Enfermagem Psiquiátrica e Ciências Humanas, Escola de Enfermagem de Ribeirão Preto da Universidade de São Paulo, Avenida dos Bandeirantes, 3900, Campus Universitário Bairro Monte Alegre, Ribeirão Preto - SP, CEP: 14040-902. e-mail: fabio.scorsolini@usp.br 